\title{
Dermatology Teachers
}

\section{DT01 \\ Barriers to and facilitators of implementation of the dermatology curriculum across UK medical schools: results of a national survey and recommendations \\ M. Sharma, ${ }^{1,2}$ R. Murphy ${ }^{1}$ and G. Doody ${ }^{1}$}

${ }^{1}$ University of Nottingham, Nottingham, UK and ${ }^{2}$ University Hospitals of Derby and Burton NHS Foundation Trust, Derby, UK

Health Education England, NHS Improvement and NHS England are working together to increase capacity, particularly in general practice (GP) and cancer services. Dermatology outpatient services align well with the training needs of the future workforce. There is lack of consistency of dermatology undergraduate (UG) curriculum implementation across UK medical schools. With $>13$ million skin-related consultations presenting to primary care annually, GP is a workforce with little UG dermatology training. To understand the barriers and facilitators for dermatology UG curriculum implementation, an online questionnaire survey aimed at UK dermatology UG teaching leads was conducted. Data were collected anonymously using mixed methodologies. The study was approved by the Nottingham Medical School Ethics committee. Seventyone per cent $(n=30 / 42)$ of UK medical schools responded to the survey. Twenty-nine responders reported to have an UG lead for dermatology at their medical school, although 11 of 30 had no formal teaching qualifications. Dermatology was a compulsory placement at 26 schools. The length of placement varied from $<5$ days (eight schools) to 5 weeks (two schools). One school had no dermatology placement. Clinical teaching occurred most frequently in secondary care consultant outpatient clinics $(n=26 ; 87 \%)$, specialty trainee clinics $(\mathrm{n}=19 ; 63 \%)$ and specialty nurse clinics $(\mathrm{n}=15 ; 50 \%)$. Twenty-six respondents (87\%) were aware of the British Association of Dermatologists' national UG curriculum, but only $13(43 \%)$ were aware if dermatology at their school was mapped to national recommendations. Over half $(n=17)$ the respondents did not feel confident in undertaking a curriculum mapping exercise. Half $(n=15)$ were unsure if dermatology assessments were blueprinted to school curricula. Over half $(\mathrm{n}=16 ; 53 \%)$ were unaware of the upcoming Medical Licensing Assessment (MLA) being introduced by the General Medical Council (GMC) across UK medical schools in 2024. Perceived barriers to curriculum implementation included demanding National Health Service commitments, dermatology not being deemed a priority in school curricula and difficulty influencing changes at medical school level. Perceived facilitators included workforce planning with support from teaching fellows and interprofessional educators. This study has shown that UG leads need time to develop and train to understand the changes in medical education such as curriculum mapping, blueprinting assessments, the role of the GMC and planned MLA. The survey suggests four broad categories to support the delivery of the dermatology curriculum nationally: (i) adequate staffing and training for UG dermatology teachers; (ii) understanding barriers to curriculum delivery at medical school level; (iii) funding/recognition of UG leadership; and (iv) addressing competing clinical pressures.

\section{DT02}

What has been the impact of COVID-19 redeployment on dermatology trainees' professional identity? An interpretative phenomenological study

\section{J. Guckian, ${ }^{1}$ N. Lee, ${ }^{2}$ J. Sutton, ${ }^{3}$ N. Myat, $^{4}$} K. Morrison, ${ }^{5}$ K. Farquhar ${ }^{6}$ and M. Singh ${ }^{7}$

${ }^{1}$ Leeds Teaching Hospitals Trust, Leeds, UK; ${ }^{2}$ Bradford Teaching Hospitals NHS Foundation Trust, Bradford, UK; ${ }^{3}$ University Hospital of Southampton NHS Trust, Southampton, UK; ${ }^{4}$ Barts and the London School of Medicine and Dentistry, London, UK; ${ }^{5}$ University of Birmingham, Birmingham, UK; ${ }^{6} \mathrm{NHS}$ Greater Glasgow and Clyde, Glasgow, UK; and ${ }^{7}$ School of Medical Sciences, Faculty of Biology, Medicine and Health, The University of Manchester, Manchester, UK

The COVID-19 pandemic has resulted in unprecedented interruptions within postgraduate dermatology training, as trainees were redeployed to medical wards for sustained periods. Junior doctors going through this transition process have expressed dread, fears of deskilling and a lack of preparedness. Professional identity formation (Goldie J. The formation of professional identity in medical students: considerations for educators. Med Teach 2012; 34: e641-8) is a dynamic process by which learners acquire a sense of self through socialization and enculturation, heavily influenced by a sense of belonging and affecting confidence and competence. Our aim was to identify the impact of COVID-19 redeployment upon dermatology trainee professional identity, factors influencing identity transitions and whether such transitions affect trainee perceptions on their future careers. Ten trainees from across the UK were purposively recruited to the study. Data were collected through semi-structured interviews and were analysed phenomenologically through the template analysis method (Brooks J. Applying qualitative research in dermatology: understanding lived experience. Br J Dermatol 2017; 177: 61718). Central and integrative themes were identified. Three central themes were identified: (i) trainee identity and values; (ii) redeployment transitions; and (iii) future clarity. Three integrative themes were found: tribes; sense of purpose; and uncertainty. A majority of trainees experienced reaffirmation of their prior values, psychologically retreating to the comfort of their close-knit communities. However, some underwent disorienting dilemmas, leading them to reflect on their futures as dermatologists. Trainees' experience of prejudicial attitudes and behaviours towards dermatology and educational 
fulfilment, driven by opportunities for reflection, supervision and staffing levels, tended to drive identity transitions. Redeployment has a strong impact on dermatology trainee professional identity and may lead to reaffirmation of prior tribal values or disorienting reflection upon future career trajectories. Improved support for community transition, reduction of uncertainty and fostering a sense of purpose among trainees may aid identity enrichment and safeguard the future dermatology consultant workforce.

\section{DT03 \\ Diversifying educational resources during the COVID-19 pandemic: delivering educational dermatology podcasts and webinars for healthcare professionals}

A. Paolino, ${ }^{1}$ A. Sears, ${ }^{1}$ R. Mills, ${ }^{1}$ J. Barker, ${ }^{1}$ F. Child,${ }^{1}$ E. Craythorne, ${ }^{1}$ F. Ferguson, ${ }^{1}$ K. Lacy, ${ }^{1}$ H. Malhomme de la Roche, ${ }^{1}$ H. McAteer, ${ }^{2}$ R. Morris-Jones, ${ }^{1}$

R. Patalay, ${ }^{1}$ E. Rashid, ${ }^{1}$ A. Salam, ${ }^{1}$ C. Smith, ${ }^{1}$ C. Stefanato, ${ }^{1}$ S. Walsh, ${ }^{3}$ L. Moorhead, ${ }^{1}$ K. Jackson ${ }^{1}$ and S. Mahil ${ }^{1}$

${ }^{1}$ Guy's and St Thomas' Hospital; ${ }^{2}$ Psoriasis Association; and ${ }^{3}$ King's College Hospital, London, UK

The COVID-19 pandemic has led to major challenges in the delivery of medical education due to social distancing rules and the redeployment of healthcare professionals to frontline specialties. 'Anytime/anywhere' learning via podcasts and webinars may address this by facilitating ongoing education for healthcare professionals (Chipps J, Brysiewicz P, Mars M. A systematic review of the effectiveness of videoconferencebased tele-education for medical and nursing education. Worldviews Evid Based Nurs 2012; 9: 78-87). Podcasts are portable digital audio files that can be streamed/downloaded to a smartphone/MP3 player and listened to at any time. Webinars feature multimedia content that can be downloaded for later viewing and for audiences located anywhere. To address the unmet need for continuing professional development in dermatology during the pandemic, we produced a series of open-access, evidence-based bespoke podcasts for clinicians on topics including facial hyperpigmentation, cutaneous manifestations of COVID-19, occupational dermatoses in the pandemic and sarcoidosis (https://www.stjohnsdermacademy.c om/podcasts). Each 20-min, peer-reviewed podcast was delivered by expert dermatologists and supplemented with online summaries of learning objectives, key facts and references. We also pioneered five educational webinars for dermatology healthcare professionals, comprising three full-day courses (Advanced Cutaneous Oncology, Dermoscopy and Specialty Certificate Examination Revision Course) and two half-day courses (Psoriasis Therapeutics Masterclass and Hidradenitis Suppurativa Masterclass; https://www.stjohnsdermacademy.c om/past-events). Educational content was delivered through a combination of live PowerPoint presentations and pre-recorded videos. Interaction with delegates was facilitated by live polling of multiple-choice quizzes and question-andanswer sessions with expert multiprofessional teaching faculties. A patient and public involvement webinar was also delivered during the pandemic, entitled 'Psoriasis, COVID-19 and Me'. To assess user experiences, we invited podcast listeners and webinar attendees to complete an online survey. Our series of podcasts received 2700 global downloads across 51 countries (including 2073 in UK, 89 in the USA and 59 in Australia). The average attendance at each webinar was 169 clinicians (range 138-199); delegates attended from a total of 27 countries. Seventy-five per cent of podcast users reported episodes as having a useful impact on their clinical practice and knowledge. Eighty-three per cent of webinar attendees rated the quality of teaching 'excellent' or 'above average'. In total, $94-99 \%$ of webinar attendees 'agreed' or 'strongly agreed' that their educational needs were met. Altogether, 75$98 \%$ of podcasts users and webinar attendees rated both the educational level and range of topics 'about right' and would recommend the resource to a colleague. Seventy-eight per cent of webinar attendees would prefer to attend future courses using an online webinar format (vs. face to face). Our experiences of delivering innovative dermatology education resources provide important shared learning opportunities. Podcasts and webinars have real potential to provide accessible, evidence-based dermatology education during the pandemic and beyond.

\section{DT04 \\ Pandemic placement: delivering the dermatology undergraduate curriculum at a UK medical school during the COVID-19 crisis \\ N. Mann, ${ }^{1}$ D. Ravindran ${ }^{1}$ and M. Sharma ${ }^{1,2}$ \\ ${ }^{1}$ Dermatology Department, University Hospitals of Derby and Burton NHS \\ Foundation Trust, Derby, UK and ${ }^{2}$ School of Medicine, University of \\ Nottingham, Nottingham, UK}

In March 2020 the first educational impact of the COVID-19 pandemic was experienced by medical students all over the UK. Owing to concerns regarding the safety of students, staff and patients, placements were suspended, immediately stopping educational opportunities. During the students' absence, dermatology staff were redeployed to support acute medical areas, dermatology services were significantly reduced and the way of working changed, including increasing use of teledermatology. There was a need for social distancing, personal protective equipment and the shielding of vulnerable staff and patients, creating challenges for students to attend placement and cover their British Association of Dermatologists' learning outcomes in the usual way. Dermatology undergraduate teaching was restructured to deliver a safe, effective and engaging placement with innovative methods. A blended learning approach was used with 1 week of e-learning and 1 week of clinical placement, in contrast to the previous 2 weeks of clinical placements. The online week - constructed to serve as a knowledge base - consisted of guided and interactive learning programmes with e-lectures, e-learning modules, videos, discussion forums and live webinars for the entire year group of 366 students. Clinical placement was adapted to maximize 